\title{
Mechanical hepaticojejunostomy: can we use a circular stapler as a viable and safe alternative? - a retrospective study of a single center
}

\author{
Nicolò Fabbri $^{1}$, Silvia Ferro ${ }^{2}$, Francesco Bagolini $^{2}$, Luigi Romeo ${ }^{2}$, Paolo Carcoforo $^{2}$, Cavallesco Giorgio ${ }^{2}$, \\ Giancarlo Pansini ${ }^{2}$ \\ ${ }^{1}$ Unit of General Surgery, Azienda USL di Ferrara, Ferrara, Italy; ${ }^{2}$ Department of Morphology, Surgery and Experimental Medicine, University of \\ Ferrara, Ferrara, Italy \\ Contributions: (I) Conception and design: N Fabbri, G Pansini; (II) Administrative support: None; (III) Provision of study materials or patients: \\ S Ferro, F Bagolini; (IV) Collection and assembly of data: S Ferro, F Bagolini; (V) Data analysis and interpretation: N Fabbri, L Romeo; (VI) \\ Manuscript writing: All authors; (VII) Final approval of manuscript: All authors. \\ Correspondence to: Nicolò Fabbri. Sant'Anna University Hospital, Via Aldo Moro 8, 44124, Ferrara, Italy. Email: nicolo.fabbri@student.unife.it.
}

Background: Since the first years of the 1980s, some authors described the use of mechanical stapler for the creation of anastomosis in biliary surgery. However, the use of these devices has not spread during the following decades, and nowadays most centers usually craft hand-sewn anastomosis.

Methods: We retrospectively collected data from medical records, surgical registries and computerized databases about the use of mechanical circular staplers for the creation of hepaticojejunostomy at our institution.

Results: From 2012 to 2020, 11 stapled hepaticojejunostomy for both neoplastic and non-neoplastic diseases were performed at our institution. The mean age of the patient was 74, with a sex distribution of 5 men and 6 women. The mean preoperative common bile duct diameter was $19 \mathrm{~mm}$. Preoperative blood samples showed mean total bilirubin of $6.95 \mathrm{mg} / \mathrm{dL}$. No intraoperative complications were reported. Two patients (18\%) had minor postoperative complications (1 wound dehiscence and 1 episode of melena that required blood transfusions), while no major complications occurred. No patients developed biliary fistula or anastomotic dehiscence. No one dies within 30 days from surgery. The mean postoperative length of stay was 13 days.

Conclusions: According to our limited experience, stapled hepaticojejunostomy seems to be a safe and effective technique in selected patients.

Keywords: Hepaticojejunostomy; biliodigestive anastomosis; mechanical stapler; pancreatoduodenectomy; jaundice

Submitted Mar 23, 2020. Accepted for publication Jul 22, 2020.

doi: 10.21037 /gs-20-387

View this article at: http://dx.doi.org/10.21037/gs-20-387

\section{Introduction}

Hepaticojejunostomy is a surgical procedure widely used in hepatopancreatobiliary (HPB) surgery that consists of the reconnection of the biliary tree to the digestive tract after its section. This surgical procedure can be considered a stand- alone treatment for some diseases such as bile duct injuries, recurrent common bile duct (CBD) stones and palliation in unresectable tumors, or can be part of a major intervention, such as pancreatoduodenectomy or liver transplant.

Classically, hand-sewn anastomosis is considered

$\wedge$ ORCID: 0000-0001-7039-3717. 
Table 1 Comorbidities per patient

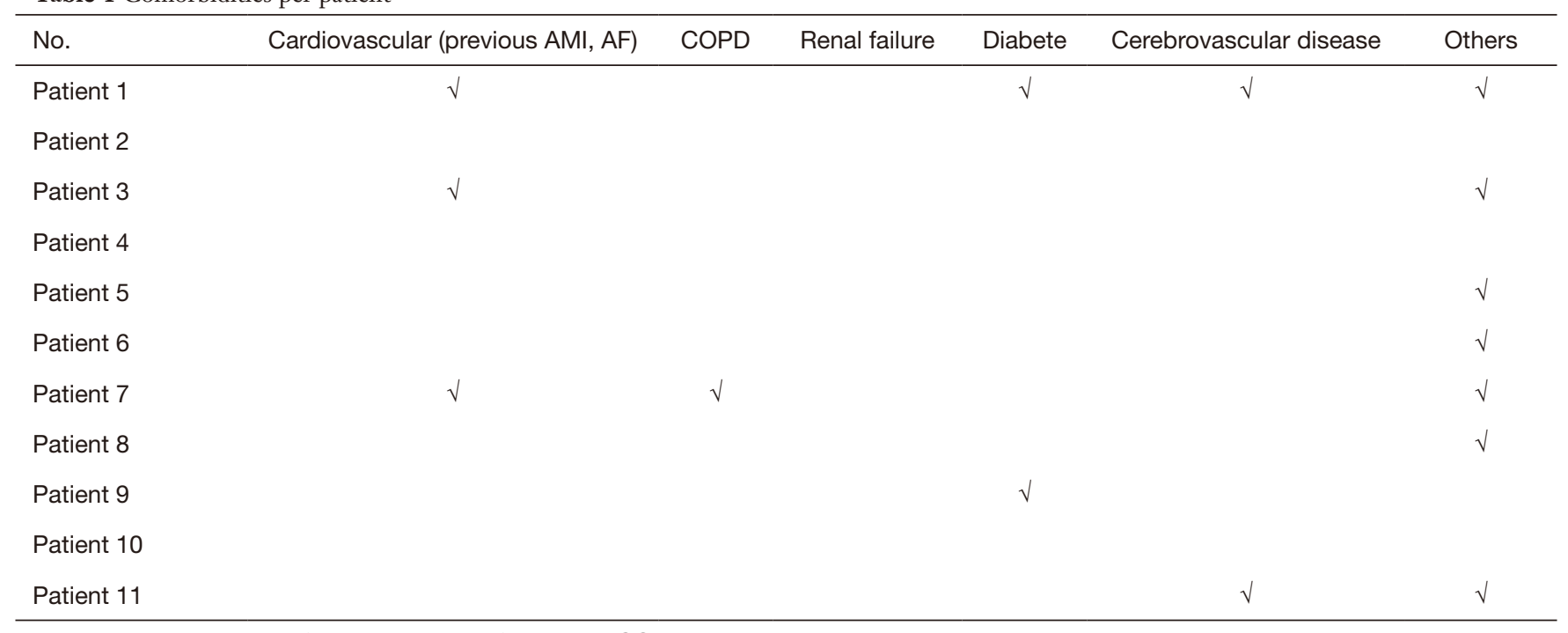

AMI, acute myocardial infarction; AF, atrial fibrillation; COPD, chronic obstructive pulmonary disease.

the standard. Different techniques for crafting hepaticojejunostomy have been described, using both continuous or interrupted sutures, and every institution that has a great experience in the surgical treatment of hepatobiliopancreatic diseases has a well-established procedure which is routinely performed. However, this standardization is not possible in those centers that treat a low number of cases requiring hepaticojejunostomy. In the last decades, the use of staplers has been introduced in HPB surgery, since Levi et al. firstly reported it in 1982 (1). The most common complications that can occur after hepaticojejunostomy are anastomotic leakage or dehiscence with biliary fistula and anastomotic stenosis. Biliary leakage is a short-term complication and can occur in $2.5-5 \%$ of cases (2), resulting in prolongation of hospital stay and need for further therapeutic procedures. Instead, anastomotic stricture, due to cicatricial stenosis or local recurrence in case of neoplastic disease is a late presenting complication, that manifests as jaundice, recurrent bile ducts lithiasis or cholangitis. Despite the large variety of diseases that can lead to performing a hepaticojejunostomy, a common trait shared by these conditions is obstructive jaundice with cholangitis, involving biliary tree dilatation due to obstruction. In the case of jaundice, the common diagnostic process starts from first-level investigations, such as abdominal ultrasound (US) and dosage of cholestasis and hepatic necrosis serum markers. Second-level investigations are radiological, such as computed tomography (CT) or magnetic resonance imaging (MRI), and endoscopic, such as esophagogastroduodenoscopy (EGDS), endoscopic ultrasounds (EUS) with biopsy in case of masses suspected for malignancy, and endoscopic retrograde cholangiopancreatography (ERCP).

We report our series of stapled biliodigestive anastomosis through a period of 8 years and analyze our results in terms of morbidity and mortality, in order to deepen the knowledge about alternative surgical techniques that can be useful especially in centers with a low HPB experience.

We present the following article in accordance with the STROBE reporting checklist (available at http://dx. doi. org/10. 21037/gs-20-387).

\section{Methods}

This study reports a retrospective analysis of patients who underwent surgical procedures that require the execution of a hepaticojejunostomy using a circular EEA stapler, for both benign and neoplastic diseases, at the Sant'Anna University Hospital of Ferrara. No exclusion criteria were considered. We collected data from medical records, surgical registries, and our institution computerized clinical database, for the period ranging from June 2012 to February 2020.

Collected data included demographic variables and comorbidity (Table 1), type of pathology (malignancy, benign diseases) (Table 2) and clinical presentation (obstructive jaundice, cholangitis, abdominal pain, loss of weight). Diagnostic and preoperative data such as blood 


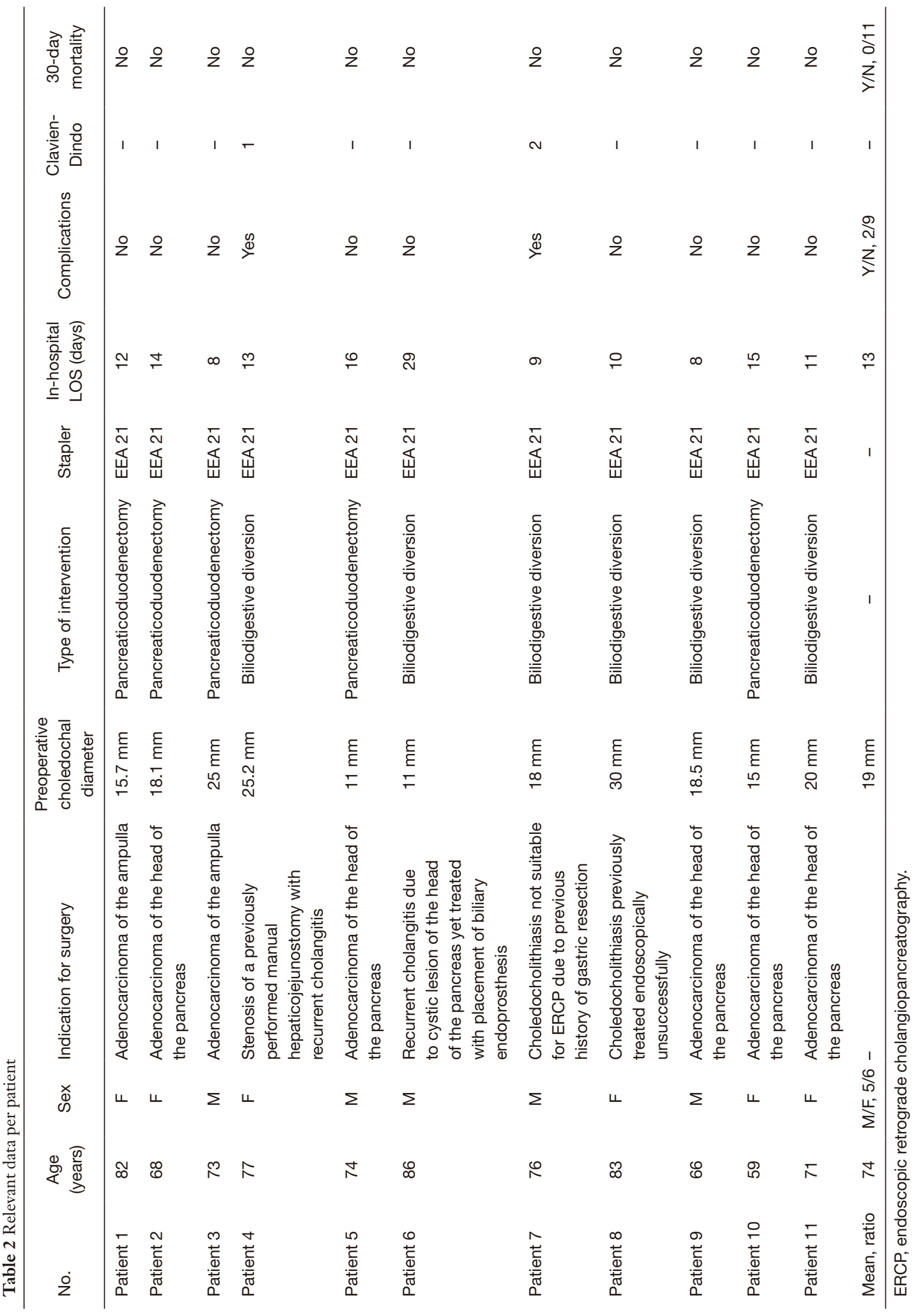




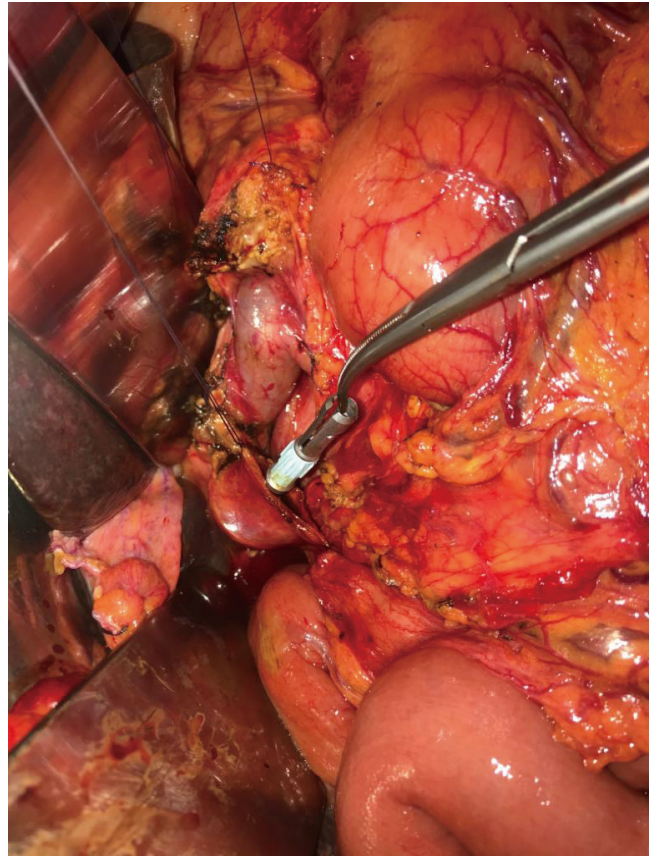

Figure 1 CEEA 21-mm stapler head placement (original image provided by Professor G. Pansini).

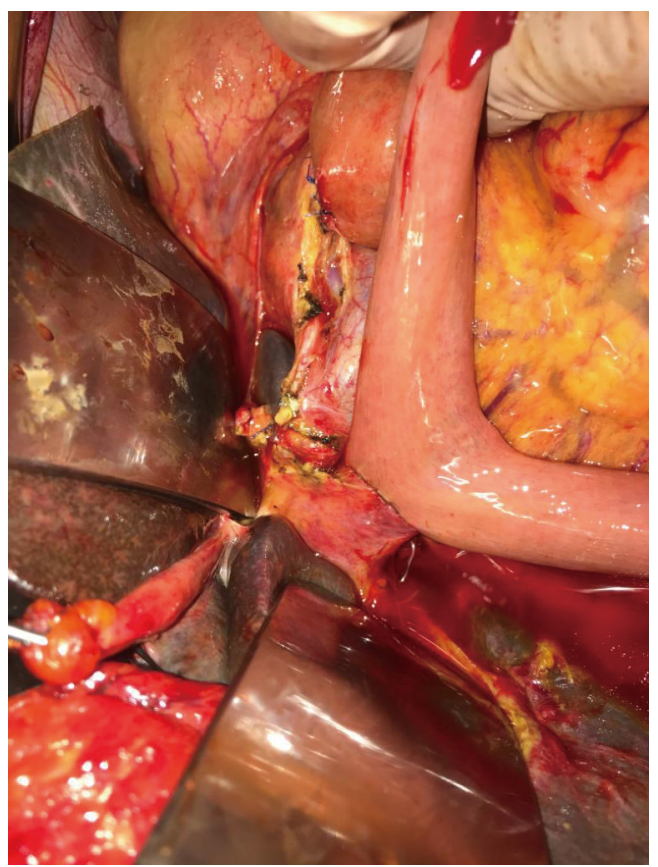

Figure 2 Bilioenteric mechanical anastomosis (original image provided by Professor G. Pansini). values, radiologic (US, CT scan, MRI) and endoscopic (EGDS, ERCP, EUS) exams were collected.

The choledochal diameter was measured from preoperative imaging (MRI or CT scans), collecting three values per patient and considering the mean of these as a plausible approximation of the real value. The need for preoperative biliary drainage, either endoscopic or percutaneous, was considered. We also collected data about the surgical procedures, such as type of intervention, the caliber of the stapler used, and intraoperative complications (defined as any complication occurred in the period ranging from anesthesia induction and postoperative awakening). As postoperative course data, we considered the length of hospital stay, major and minor post-operative short-term complications, development of anastomotic leakage or dehiscence, and 30-day mortality. We considered short-term complications that occurred within 30 days after surgery. Cardiovascular accident, Cerebrovascular events, Thromboembolism are considered as major complications. We report qualitative variables as a percentage, while continue ones are reported as mean \pm standard deviation (SD) (range). The study was conducted in accordance with the Declaration of Helsinki (as revised in 2013). The study was approved by regional ethics committee of Our Institution (NO.: 837/2019/ Oss/AOUFe) and individual consent for this retrospective analysis was waived.

\section{Surgical technique}

After isolation and section of the CBD, the surgeon places a purse-string suture around the proximal portion of the CBD and inserts the anvil of the mechanical stapler in the lumen of the CBD (Figures 1-3). Then he closes the lumen tying the suture (Figure 4). After that, a jejunal loop is transposed in the supramesocolic lodge, passing through the transverse mesocolon, according to the Roux-en-Y technique. The body of the circular stapler is inserted in the transposed jejunal loop through its proximal end, were the jejunum were transected before its transposition. The surgeon extracts the trocar of the stapler, piercing the intestinal wall, and connect it with the anvil. After the retraction of the trocar, the surgeon fires the stapler, completing the anastomosis. 


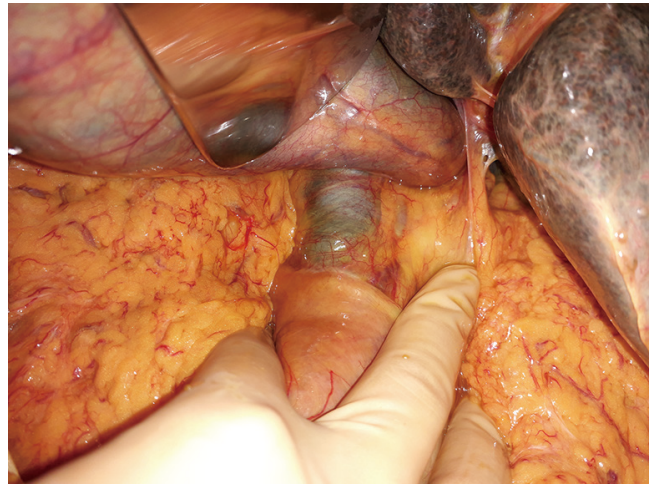

Figure 3 Dilatation of the main biliary tract (original image provided by Professor G. Pansini).

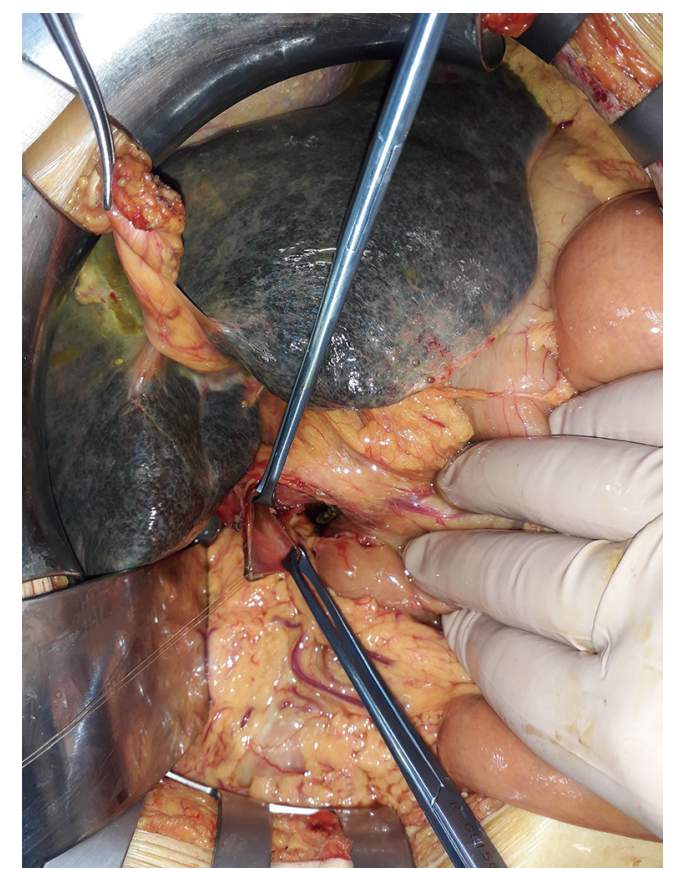

Figure 4 Exploration of the lumen of the main biliary tract (original image provided by Professor G. Pansini).

\section{Results}

During the examined period, hepaticojejunostomy crafted using a circular EEA stapler, according to the abovementioned technique, were performed in 11 patients, 5 men, and 6 women, while the mean age was 74 (range, 59-86) years. Table 1 shows baseline characteristics for each patient. Among these patients, 7 were affected by adenocarcinoma of the pancreatic head or of the ampulla, while the remnant 4 patients were affected by non-neoplastic diseases. All data were available for all patients.

Table 2 shows relevant data for each patient.

Preoperative radiological investigations were distributed as follows: 9 patients underwent the abdominal US, 9 patients underwent contrast-enhanced abdominal CT scan, 4 patients underwent MRI. All patients underwent at least one imaging between the abdominal CT scan and MRI. Six patients underwent EGDS, but only 4 ERCP was performed. In one case ERCP was not performed due to the presence of duodenal neoplastic stenosis that makes its execution impossible. Only one case underwent endoscopic biliary drainage with $10 \mathrm{~cm} \times$ 7 Fr plastic stent.

The mean preoperative CBD diameter was 19 (range, 11-30) mm. Mean preoperative total bilirubin $6.95 \mathrm{mg} / \mathrm{dL} .6$ patients affected by cephalopancreatic neoplasms underwent pancreatoduodenectomy with curative intent, in only one case of neoplastic disease an intervention with palliative intent (biliodigestive diversion) was performed, For the other four cases, a simple biliodigestive diversion was performed. All hepaticojejunostomy was performed using a 21-mm EEA stapler in all cases. No intraoperative complications or malfunction of the device happened.

No anastomotic leak or dehiscence occurred, such as major complications and reinterventions. Two patients developed minor complications: 1 patient presented wound dehiscence of skin and subcutaneous fat with conservation of the integrity of the fascial layer (Clavien-Dindo grade I), and 1 patient presented an episode of melena that required blood transfusions and no other therapeutic procedure (Clavien-Dindo grade II). Seven patients were supported with blood transfusions after surgery, without any sign of active bleeding. One patient received only transfusions of plasma. The remnant 3 patients did not require any postoperative transfusions.

The mean postoperative length of stay (LOS) was 13 days. No one died within 30 days after surgery.

One patient died 2 months after surgery, for an event not related to surgical intervention. Of the other patients, 4 died at the time of data collection, 15, 16 and 24 months after surgery respectively for the development of the neoplastic disease. Lastly, in one case was impossible to establish the date of death. None of the patients developed jaundice or cholangitis due to anastomotic stenosis. The mean survival rate in dead patients was 402.5 (range, 80-725) days. To date, the longest survival of an operated patient is 1,025 days, for a non-cancerous disease. 


\section{Discussion}

Manual hepaticojejunostomy is classically considered the preferred technique for reconnection of the biliary tree to the digestive tract. The adopted technique for this anastomosis mainly depends on the "surgical school" and expertise of the operating surgeon and can be performed with either continuous or interrupted sutures (2).

After the introduction of a surgical stapler for general surgery, a few experiences shed light on the possibility of a new surgical approach. The earliest mention of the use of a mechanical stapler in HPB surgery is in 1984 by Levi (1), who reported 18 cases of triangle-shaped Roux-en-Y cholecystojejunostomy using TA stapler with palliative intent. The following year, Mullin et al. (3) reported three cases of cholecystojejunostomy using circular EEA stapler for palliation of obstructive jaundice in unresectable pancreatic cancer. After these first reports, many authors reported isolated experiences with stapled cholecystojejunostomy, but it was not until 1999 that the first case of stapled choledochojejunostomy with circular stapler was reported (4).

Despite the used technique, biliodigestive anastomosis is burdened by short-term and long-term complications. The most relevant short-term complication of hepaticojejunostomy is biliary fistula due to anastomotic leakage. The reported incidence may vary from $2 \%$ to $8 \%$ according to different series $(5,6)$. Biliary fistula can lead to prolonged length of hospital stay and additional diagnostic and therapeutic procedures, which increase the cost for the healthcare providers and morbidity for patients. Anastomotic stenosis occurs in about $3.5-8 \%$ of hepaticojejunostomy. Usually, the cause of anastomotic stenosis is scar tissue or local recurrence of neoplastic disease. Its clinical presentation can be with jaundice, biliary stones or recurrent cholangitis due to reduced flow of bile through the biliary ducts. To our knowledge, the largest case series available in literature before the one we report is that reported by Tersigni et al. (7). They successfully completed seven stapled hepaticojejunostomy in as many pancreaticoduodenectomies, reporting a regular postoperative course, without any complications. Only one patient developed an episode of cholangitis 8 months after surgery and MRI showed a biliodigestive anastomosis with a diameter of $10 \mathrm{~mm}$. Another patient died 6 months after surgery. The remaining patients did not complain about symptoms that could be attributed to anastomotic stenosis and showed a good anastomosis diameter at 1-year follow-up imaging.

In our small sample, none of the patients developed biliary fistula as postoperative complications. Only two patients developed postoperative complications (grade I and grade II according to Clavien-Dindo classification), not surely related to the use of mechanical stapler and without impact on long-term outcomes. None of the patients developed symptoms related to anastomotic stricture.

The patients included in this report underwent many diagnostic procedures, which includes at least one between the CT scan and MRI. These procedures can give an estimate of the biliary tract dilatation, which is essential to consider if the surgeon plan a stapled biliodigestive anastomosis. Previous experiences reported a bile duct diameter of $20 \mathrm{~mm}$ as a necessary condition. We report a mean CBD diameter of $19 \mathrm{~mm}$ at preoperative imaging evaluation. This result is in line with the literature. In fact, the smallest circular stapler available is $21 \mathrm{~mm}$ in diameter. This result reflects the need for a bile duct large enough to place the stapler anvil.

Our experience, consisting of 11 cases, shows that, in selected cases, this technique is not harmful to the patient, as only one of the subjects suffered major complications associated with the procedure, but not directly associated with the biliary anastomosis. Despite the small number of cases reported in the literature, in our experience using a mechanical stapler to fashion hepaticojejunostomy seems to be a promising alternative to the manual technique in selected patients with biliary tract dilatation that allow the introduction of the anvil in the biliary stump. In recent years, the surgical community raised many doubts about preoperative biliary drainage for jaundice due to resectable pancreatic cancer. This new trend allows for the more dilated biliary tract at the time of surgery, which could facilitate wider use of staplers for hepaticojejunostomy. Mechanical stapler used $(21 \mathrm{~mm})$ is a little different to larger models; in fact, the stapler offers a favorable angle for the introduction in the bowel and to align the intestine with the shorter bile duct. Moreover, the use of staplers could potentially reduce the operatory time if compared with hand-sewn anastomosis and allow less experienced surgeons to perform such a critical step of many surgical procedures.

\section{Conclusions}

This report presents many pitfalls, as it is a simple case series. In fact, we did not perform any comparison with other techniques for hepaticojejunostomy fashioning and 
the number of cases reported is limited, so no definitive conclusions can be done. However, we hope that these results will encourage further researches, in order to confirm or confute them in a wider sample.

\section{Acknowledgments}

Funding: None.

\section{Footnote}

Reporting Checklist: The authors have completed the STROBE reporting checklist. Available at http://dx. doi. org/10. 21037/gs-20-387

Data Sharing Statement: Available at http://dx. doi. org/10.21037/gs-20-387

Conflicts of Interest: All authors have completed the ICMJE uniform disclosure form (available at http://dx. doi. org/10.21037/gs-20-387). The authors have no conflicts of interest to declare.

Ethical Statement: The authors are accountable for all aspects of the work in ensuring that questions related to the accuracy or integrity of any part of the work are appropriately investigated and resolved. The study was conducted in accordance with the Declaration of Helsinki (as revised in 2013). The study was approved by regional ethics committee of Our Institution (NO.: 837/2019/Oss/AOUFe) and individual consent for this retrospective analysis was waived.

Open Access Statement: This is an Open Access article

Cite this article as: Fabbri N, Ferro S, Bagolini F, Romeo L, Carcoforo P, Giorgio C, Pansini G. Mechanical hepaticojejunostomy: can we use a circular stapler as a viable and safe alternative? - a retrospective study of a single center. Gland Surg 2020;9(5):1298-1304. doi: 10.21037/gs-20-387 distributed in accordance with the Creative Commons Attribution-NonCommercial-NoDerivs 4.0 International License (CC BY-NC-ND 4.0), which permits the noncommercial replication and distribution of the article with the strict proviso that no changes or edits are made and the original work is properly cited (including links to both the formal publication through the relevant DOI and the license). See: https://creativecommons.org/licenses/by-nc-nd/4.0/.

\section{References}

1. Levi JU, Zeppa R, Hutson D, et al. A rapid technique for biliary and duodenal bypass in nonresectable pancreatic carcinoma. Arch Surg 1982;117:375-6.

2. Brunner M, Stockheim J, Krautz C, et al. Continuous or interrupted suture technique for hepaticojejunostomy? A national survey. BMC Surg 2018;18:84.

3. Mullin TJ, Damazo F, Dawe EJ. Cholecystoenteric anastomosis with the EEA stapler for cancer of the pancreas. Am J Surg 1983;145:338-42.

4. Cirocchi R, Covarelli P, Mazieri M, et al. Choledochojejunostomy using a mechanical stapler. Chir Ital 1999;51:177-9.

5. Antolovic D, Koch M, Galindo L, et al. Hepaticojejunostomy--analysis of risk factors for postoperative bile leaks and surgical complications. J Gastrointest Surg 2007;11:555-61.

6. Kadaba RS, Bowers KA, Khorsandi S, et al. Complications of biliary-enteric anastomoses. Ann R Coll Surg Engl 2017;99:210-5.

7. Tersigni R, Capaldi M, Cortese A. Biliodigestive anastomosis with circular mechanical device after pancreatoduodenectomy: our experience. Updates Surg 2011;63:253-7. 